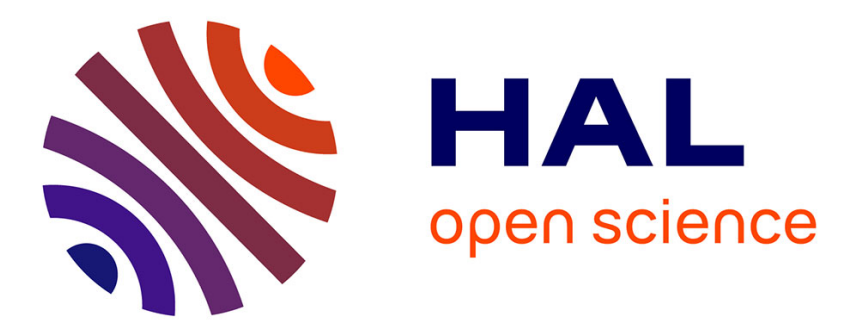

\title{
Passive tomography by elastic guided wave for corrosion detection in pipelines
}

Huu Tinh Hoang, Tom Druet, Bastien Chapuis, Emmanuel Moulin

\section{To cite this version:}

Huu Tinh Hoang, Tom Druet, Bastien Chapuis, Emmanuel Moulin. Passive tomography by elastic guided wave for corrosion detection in pipelines. IWSHM 2019 - The 12th International Workshop on Structural Health Monitoring, Sep 2019, Stanford, United States. cea-03251430

\section{HAL Id: cea-03251430 https://hal-cea.archives-ouvertes.fr/cea-03251430}

Submitted on 7 Jun 2021

HAL is a multi-disciplinary open access archive for the deposit and dissemination of scientific research documents, whether they are published or not. The documents may come from teaching and research institutions in France or abroad, or from public or private research centers.
L'archive ouverte pluridisciplinaire HAL, est destinée au dépôt et à la diffusion de documents scientifiques de niveau recherche, publiés ou non, émanant des établissements d'enseignement et de recherche français ou étrangers, des laboratoires publics ou privés. 


\title{
Passive Tomography by Elastic Guided Wave for Corrosion Detection in Pipelines
}

\section{HUU TINH HOANG, TOM DRUET, BASTIEN CHAPUIS and EMMANUEL MOULIN}

\begin{abstract}
Corrosion presents a major challenge for various industries, especially for petrochemical and nuclear industry. Estimating accurate wall thickness maps of pipes or pressure vessels is of great importance for detecting corrosion damage in these structures and assessing its remaining lifetime. Guided wave tomography provides a solution for this problem in which pipe-like structures have a high diameter to thickness ratio, by sending guided waves through the region of interest, then using tomographic imaging $[1,2]$ to reconstruct the thickness map, significantly eliminating the need to take measurements at all points across the surface. The Structural Health Monitoring (SHM) process involves the observation of a structure over time by using measurements from sensors embedded in this structure in order to monitor its current state of health and hence taking at the right time corrective action to prevent further rupture or leakage. It is essential to use a small number of sensors to limit the intrusiveness of SHM system. Consequently, regularization is applied to adapt tomography in the context of the SHM system. The tomographic algorithm is evaluated in experiments. We show that this tomographic algorithm is particularly well adapted to a so-called "passive" solution, where the ambient elastic noise which naturally presents in an operating structure (due to vibrations, aerodynamic turbulence) is exploited to make tomography by cross correlation [3], no need the emission of waves by the system. The complexity of the embedded SHM system is therefore reduced.
\end{abstract}

\section{INTRODUCTION}

Corrosion and erosion damage are major causes of pipeline failure. Continuous monitoring of corrosion damage in pipelines plays a central role in revealing unforeseeable changes which could have developed during its lifetime. The Structural Health Monitoring (SHM) process involves the observation of a structure over time by using measurements from sensors embedded in this structure in order to monitor its current state of health and hence taking at the right time corrective action to prevent further rupture or

Huu Tinh Hoang, Tom Druet, Bastien Chapuis, CEA LIST, F-91191 Gif-sur-Yvette, France. Email: huutinh.hoang@cea.fr

Emmanuel Moulin, Polytechnic University Hauts de France, France 
leakage. Ultrasonic guided waves, the acoustic waves that can propagate a long distance with little loss in energy along an elongated structure while guided by its boundaries, is a potential physical investigation means for our SHM system. In cases of severe wall loss over a large area coverage, guided wave tomography (GWT) has demonstrated the capacity of detection, localization and characterization of corrosion damage $[1,4]$. However, these innovative methods are difficult to implement in harsh environments (at high temperatures or even in a radioactive environment) for which the conventionally used sensors (piezoelectric ceramics) are not sufficiently resistant. In such environments, it is possible to use optical fibers provided with Bragg gratings [5-7], which can serve as ultrasonic wave receivers, but not transmitters. In order to get rid of the "active" source, we propose using the so-called "passive" methods based on the correlation of the diffuse elastic field. These methods are from geophysics $[8,9]$ and have recently made significant advances in this field. They are based on the analysis of waves which naturally present in the structure, often assimilated to noise. In an industrial structure, the exploitable noise sources can be, for example, a turbulent fluid in a pipeline, aerodynamic turbulence on the fuselage of an aircraft, vibrations due to engines or reactors or waves on the hull of a boat. Specifically, this paper will aim to develop and exploit experimental benches using these diffuse elastic field correlation methods in order to extract significant parameters on the health status of metallic pipes delimited by two rings of ultrasonic transducers as shown in Figure 1a.

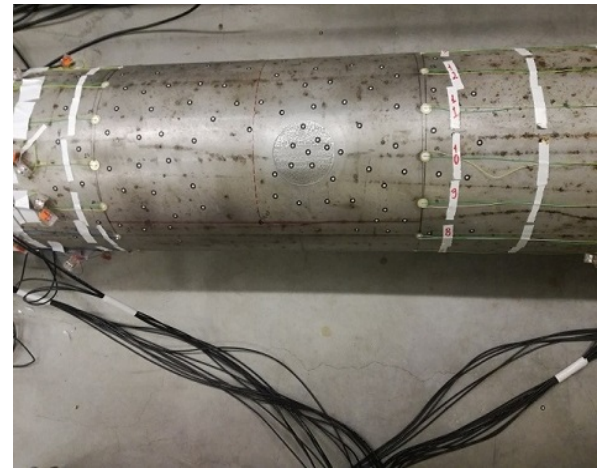

(a)

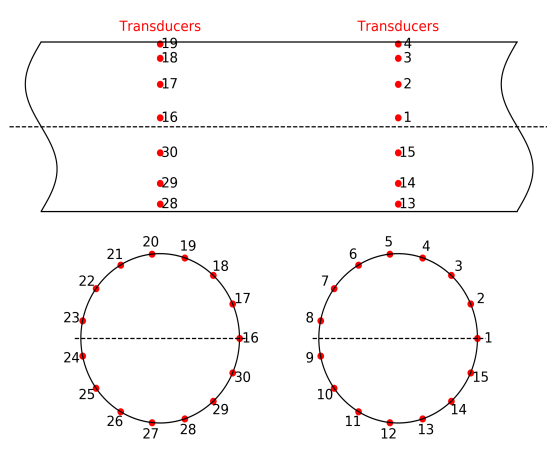

(b)

Figure 1: Example of monitoring configuration for straight pipe with a defect of corrosion. (a) Overall configuration . (b) Diagram showing transducer position and numbering.

In the following section, passive tomography by guided waves is described, including diffraction tomography algorithm, the passive method, the array system and data acquisition and the data processing method. The next section is the results of active and passive tomography for corrosion detection in pipeline systems. Discussions are followed and conclusions are summarized in the final section.

\section{METHODS}

\section{Tomography by guided waves in pipelines}


The scattering model used is similar to the formulations of [2]. The acoustic model is derived assuming that the velocity of a guided wave at a point in the pipe is dependent only on the thickness of the pipe at that point. In addition, it is assumed that the wall thickness is small compared with the pipe radius and therefore that guided wave propagation and scattering can be approximated by unwrapping the pipe and treating it as a flat plate with the same wall thickness. In the frequency domain, the wave equation is expressed as the Helmholtz equation

$$
\left[\nabla^{2}+k(x)^{2}\right] \phi=0
$$

where $k(x)$ represents the local wavenumber at position $x$ and $\phi$ is scalar field potential. Defining an object function

$$
O(x)=k_{0}^{2}\left[\left(\frac{c_{0}}{c(x)}\right)^{2}-1\right]
$$

where $c(x)$ represents the local phase velocity at position $x, k_{0}$ and $c_{0}$ is the background wavenumber and phase velocity in the undamaged domain with uniform thickness, equation (1) can be rearranged to give

$$
\left[\nabla^{2}+k_{0}^{2}\right] \phi=-O \phi
$$

Taking the free space Helmholtz equation

$$
\left[\nabla^{2}+k_{0}^{2}\right] \phi_{0}=0
$$

this can then be subtracted from (3) to give an equation in terms of the scattered field $\phi_{s}=\phi-\phi_{0}$,

$$
\left[\nabla^{2}+k_{0}^{2}\right] \phi_{s}=-O \phi
$$

The scattered field can be obtained under an integral formulation by Lippmann-Schwinger equation

$$
\phi_{s}=-\int O(x) \phi(x) G(x, y) d x
$$

where the Green's function $G(x, y)$ is an elemental solution to (5) for a delta function source at position $x$ and measurement location at $y$. As described in [2], equation (6) can be formulated in the near field as

$$
\phi_{s}(z, y)=-\int O(x) G(z, x) G(x, y) d x,
$$

for a source at $z$ and a receiver at $y$, assuming that the sources are delta functions and hence produce incident fields corresponding to Green's function. To perform the inversion, we use the formulation described in [2]

$$
O(x)=-\int_{-\pi}^{\pi} \int_{-\pi}^{\pi} \frac{\phi_{s}}{G(z, x) G(x, y)} W d s_{\theta} d r_{\theta},
$$

where $s_{\theta}$ and $r_{\theta}$ represent the angles of the incident and scattered directions, respectively. Note that the weighting function

$$
W=k_{0}\left|\sin \Delta_{\theta}\right|,
$$


where $\Delta_{\theta}=s_{\theta}-r_{\theta}$, is the term which essentially differentiates beamforming from diffraction tomography (DT).

\section{Passive Method for detection of Corrosion}

The principle of the passive method is to use the correlation of the field, and not the field itself, to reconstruct the information of propagation between points to investigate the area bounded by these points. Passive method can exploit the presence of ambient noise in the structure generated by, for example, the circulation of fluids in pipeline systems or aerodynamic turbulences.

Indeed, the relationship between the cross-correlation and the Green's function has been established in $[10,11]$. One can create data for tomography from random field by correlating records from different locations. Green's function is extracted by the following expression:

$$
G\left(x_{A}, x_{B}, t\right)+G\left(x_{A}, x_{B},-t\right) \propto \frac{1}{T} \int_{0}^{T} \varphi\left(x_{A}, \tau\right) \varphi\left(x_{B}, \tau-t\right) d \tau,
$$

where $\varphi\left(x_{A}, \tau\right)$ and $\varphi\left(x_{B}, \tau\right)$ are random wave fields measured at $x_{A}$ and $x_{B}$.

\section{Working Configuration}

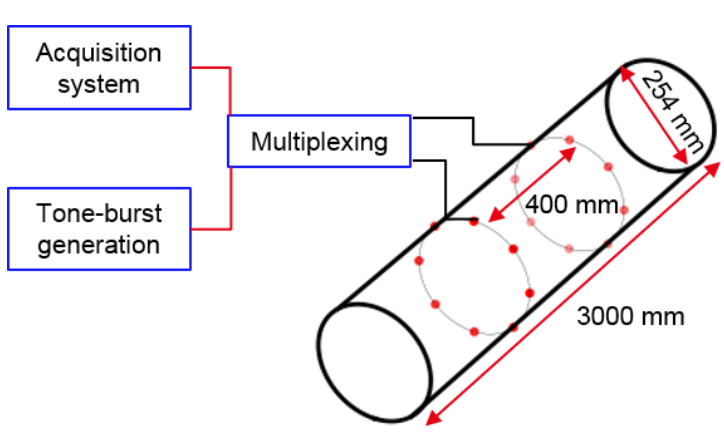

(a)

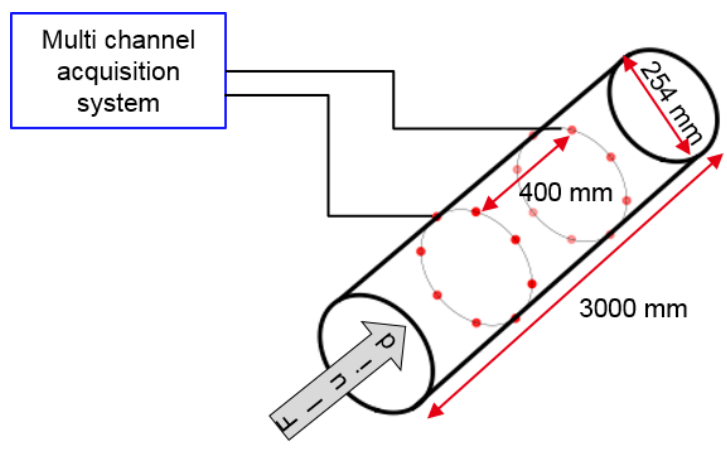

(b)

Figure 2: Schematic views of working configuration for data acquisition. (a) Active configuration . (b) Passive configuration.

Figure 2 illustrates the experimental setup for data acquisition for active and passive tomography. To measure the guided wave signals, two ring arrays of 15 piezoelectric sensors were used (see Figure 1b). In the active case, the piezoelectric transducers were excited by a five-cycle Hanning windowed toneburst centered at $40 \mathrm{kHz}$, which corresponds to a wavelength $22 \mathrm{~mm}$. Figure 3 a shows a typical set of signals received by all 15 receivers in a ring array of sensors when transmitting with one of the piezoelectric transducer in the other ring array. In the passive case, 20 seconds of noise generated by sweeping a jet of compressed air inside the pipe were recorded by the piezoelectric sensors. For the same sensor pairs in the active set, the filtered cross-correlation signals at $40 \mathrm{kHz}$ are shown in the Figure $3 \mathrm{~b}$. As the time interval is the same, the comparison shows a good agreement for the first wave packets between two cases. 


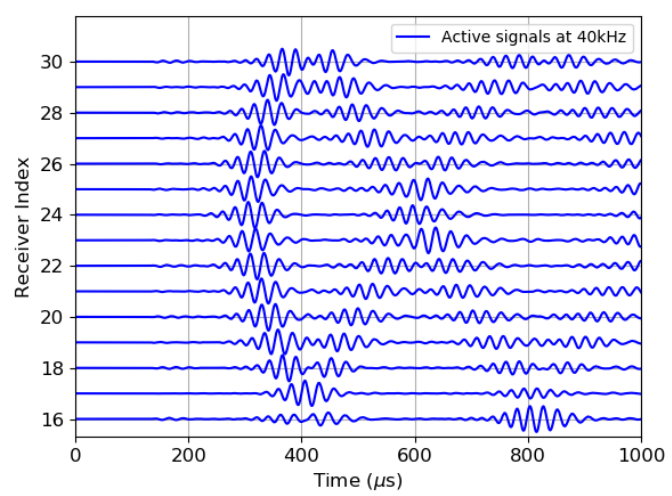

(a)

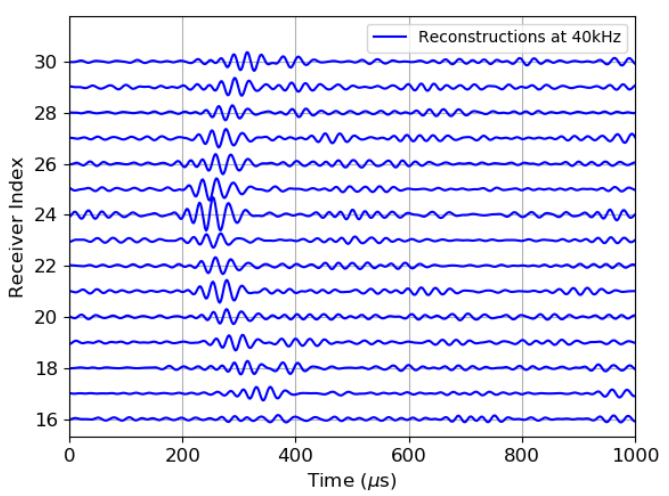

(b)

Figure 3: Ultrasonic signals. (a) Active signals. (b) Passive reconstructions.

\section{Signal Processing}

The aim of signal processing is to calibrate the measured data obtained from experiments with simple acoustic modeling data used in the forward modeling described in the previous section, so that the approximation made in the wave propagation and inversion algorithm is taken into account. Firstly, the frequency domain data needed for the inversion were obtained by applying a fast Fourier transform to the time-traces from each send-receive pair and then extracting the frequency component(s) required. The calibration factor for inversion data was determined as [12] by calculating the ratio of homogeneous Green's function $G_{0}$ and transformed data of experiment $\phi_{0}$ when no defect presents in inspection section

$$
c^{i j}=\frac{G_{0}^{i j}}{\phi_{0}^{i j}},
$$

where $i$ and $j$ represent the numberings of emitter and receiver, respectively. This factor is then used to calibrate the total field $\phi_{i j}$ of experiment of the corresponding emitterreceiver pair $i j$ to give the inputs data in the diffraction tomography

$$
\phi_{\text {calibrated }}^{i j}=c^{i j} \phi_{i j} .
$$

Inversion is also regularized by Gaussian smoothing of the perturbation model to minimize high-frequency artifacts.

After the inversion by (8) with the calibrated data of active signals or passive reconstructions from noise, the reconstructed velocity maps are converted to thickness maps by the known dispersion relationship between the thickness, frequency, and phase velocity.

\section{EXPERIMENTAL RESULTS}

\section{Active tomography for corrosion detection}


Firstly, the thickness reconstruction map by active guided wave tomography is introduced in Figure 4a compared with the reference image measured with a 3D scanner as shown in Figure $4 \mathrm{~b}$. The position of corrosion defect is detected. Although the small

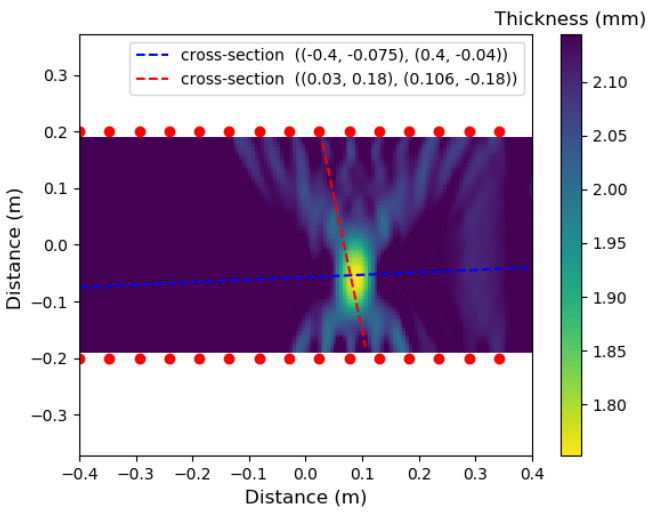

(a)

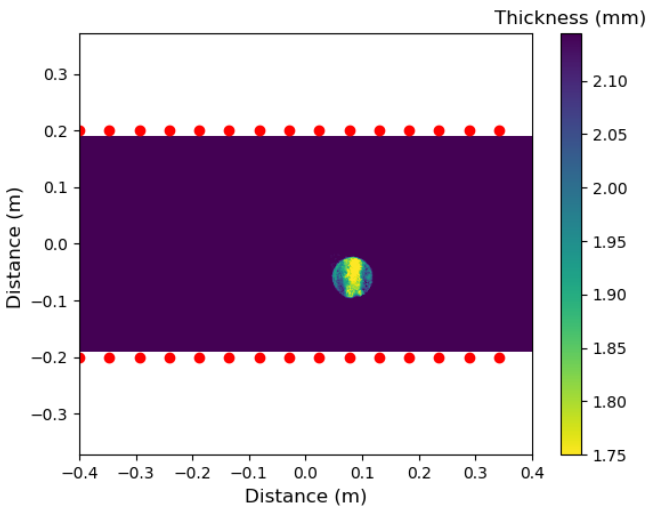

(b)

Figure 4: Active tomography by guided wave. (a) Thickness reconstruction. (b) Reference by a 3D scanner.

spatial variations are not well reconstructed because of the algorithmic resolution, the minimal remaining thickness is estimated according to the two cross-sections of Figure 4 shown in Figure 5. The blue dashed curve - Figure 5a - is lateral cross-section corresponding to the blue dashed line - Figure $4 \mathrm{a}$ - and the red dashed one - Figure $5 \mathrm{~b}$ - is axial cross-section corresponding to the red dashed line - Figure 4a. The lateral resolution is expected to be better than the axial one because of the lack of sensors (denoted by red dots) along pipe axis as shown in Figure 5.

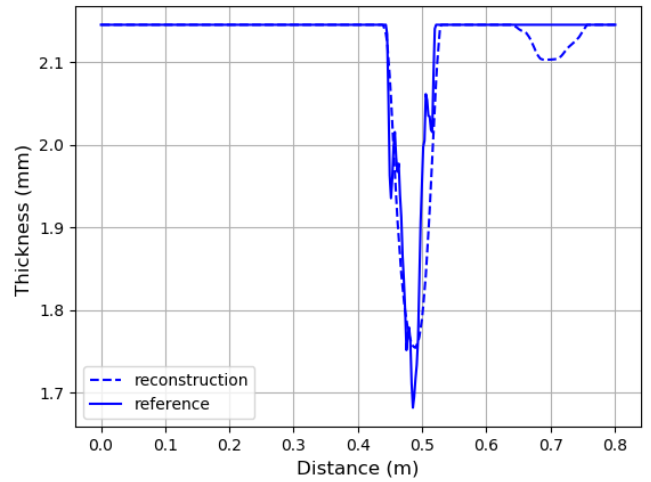

(a)

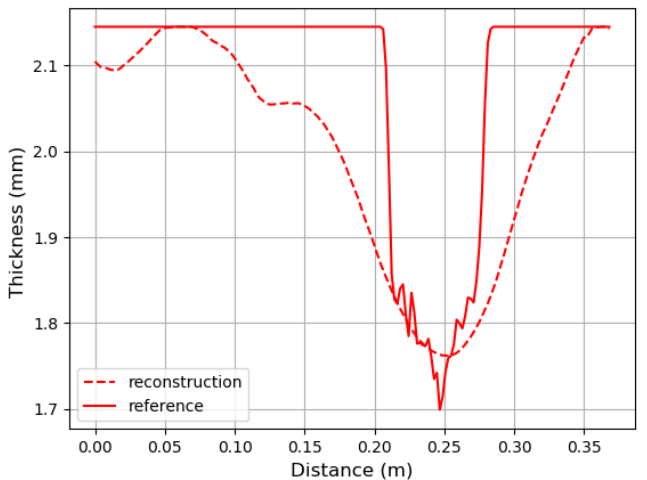

(b)

Figure 5: Cross-sections of Figure 4. (a) Lateral cross-section. (b) Axial cross-section.

\section{Passive tomography for corrosion detection}

Secondly, the thickness reconstruction map of the same pipe section (and same corrosion defect) is shown in Figure 6 a by passive guided wave tomography with the filtered 
cross-correlation of noise (which was generated by sweeping a jet of compressed air inside the pipe, as described in the working configuration section) at $40 \mathrm{kHz}$ as input data. Although some artifacts degrade the quality of image, it can be seen from this figure that the defect was reconstructed and localized. Comparison between the reference thickness (see Figure $4 \mathrm{~b}$ ) and the lateral reconstruction profile across the defect extracted from Figure $6 \mathrm{a}$ are shown in Figure $6 \mathrm{~b}$. The reconstruction from acquired noise in passive case underestimates the depth by $0.2 \mathrm{~mm}$. As in the calibration, the acquired noise was generated by an uncontrollable source (a jet of compressed air), and therefore, this mismatch in noise power between the case without defect and with defect leads to the amplitude errors in the inversion.

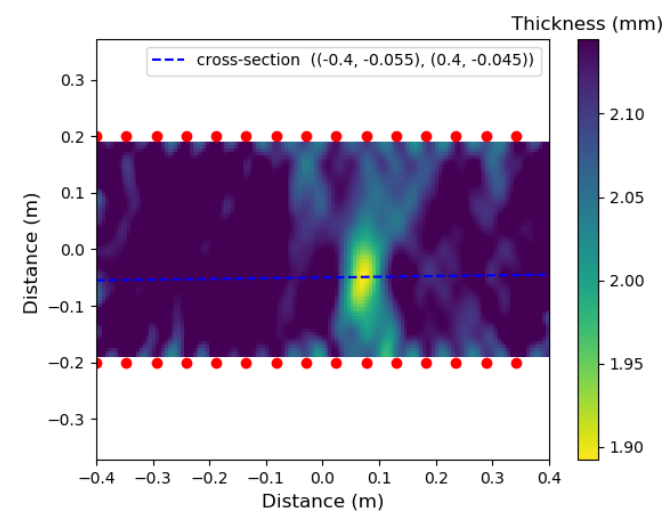

(a)

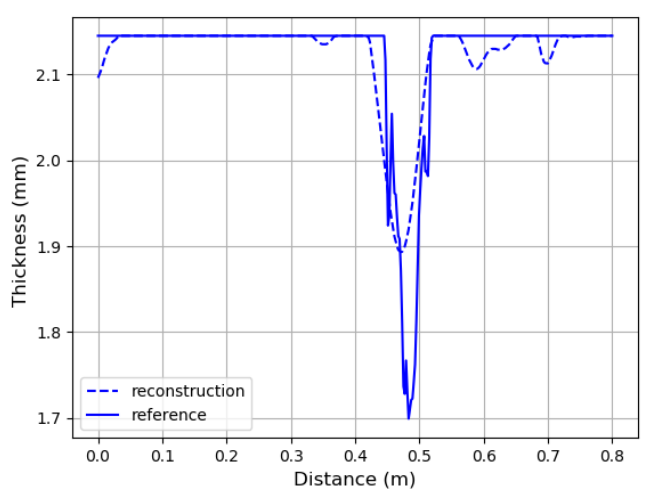

(b)

Figure 6: Passive tomography by guided wave. (a) Passive thickness reconstruction. (b) Lateral cross-section.

\section{CONCLUDING REMARKS}

In this paper, the active and passive tomography for corrosion detection and characterization have been presented and analyzed. The obtained results in both cases have a good agreement in terms of detection and location a corrosion defect. In the future works, the high-resolution guided wave tomography algorithm [12] will be integrated to improve the thickness reconstruction and the autocalibration method [13] will be tested to resolve in particular the problem of uncontrollable sources of noise in passive tomography and in general the baseline-free monitoring problem in the SHM system.

Additionally, the piezoelectric transducers are replaced by optical fiber Bragg grating sensors in the next step to reduce the intrusiveness of sensors in the SHM system. Some obtained results of active hybrid tomography using one ring of piezoelectric transducers and one ring of fiber Bragg grating sensors are presented in [14]. Those encouraging results then lead to a long-term goal, like implementing the passive tomography with only fiber Bragg grating sensors for nuclear application in which the temperature will be very high. 


\section{REFERENCES}

1. Huthwaite, P. 2014. "Evaluation of inversion approaches for guided wave thickness mapping," Proceedings of the Royal Society A: Mathematical, Physical and Engineering Sciences, 470(2166):20140063-20140063, doi:10.1098/rspa.2014.0063.

2. Huthwaite, P. 2016. "Eliminating incident subtraction in diffraction tomography," Proceedings of the Royal Society A: Mathematical, Physical and Engineering Science, 472(2195):20160707, doi:10.1098/rspa.2016.0707.

3. Moulin, E., N. A. Leyla, J. Assaad, and S. Grondel. 2009. "Applicability of acoustic noise correlation for structural health monitoring in nondiffuse field conditions," Applied Physics Letters, 95(9):094104, doi:10.1063/1.3200240.

4. Huthwaite, P. 2016. "Improving accuracy through density correction in guided wave tomography," Proceedings of the Royal Society A: Mathematical, Physical and Engineering Science, 472(2186):20150832, doi:10.1098/rspa.2015.0832.

5. Lee, J.-R., S.-S. Lee, and D.-J. Yoon. 2008. "Simultaneous multipoint acoustic emission sensing using fibre acoustic wave grating sensors with identical spectrum," Journal of Optics A: Pure and Applied Optics, 10(8):085307, doi:10.1088/1464-4258/10/8/085307.

6. Pang, D. and Q. Sui. 2014. "Response analysis of ultrasonic sensing system based on fiber Bragg gratings of different lengths," Photonic Sensors, 4(3):281-288, doi:10.1007/s13320014-0157-4.

7. Druet, T., B. Chapuis, M. Jules, G. Laffont, and E. Moulin. 2018. "Passive guided waves measurements using fiber Bragg gratings sensors," The Journal of the Acoustical Society of America, 144(3):1198-1202, doi:10.1121/1.5054015.

8. Bensen, G. D., M. H. Ritzwoller, M. P. Barmin, A. L. Levshin, F. Lin, M. P. Moschetti, N. M. Shapiro, and Y. Yang. 2007. "Processing seismic ambient noise data to obtain reliable broad-band surface wave dispersion measurements," Geophysical Journal International, 169(3):1239-1260, doi:10.1111/j.1365-246x.2007.03374.x.

9. Chen, K.-X., Y. Gung, B.-Y. Kuo, and T.-Y. Huang. 2018. "Crustal Magmatism and Deformation Fabrics in Northeast Japan Revealed by Ambient Noise Tomography," Journal of Geophysical Research: Solid Earth, 123(10):8891-8906, doi:10.1029/2017jb015209.

10. Sabra, K. G. 2005. "Extracting time-domain Green's function estimates from ambient seismic noise," Geophysical Research Letters, 32(3), doi:10.1029/2004g1021862.

11. Sabra, K. G., E. S. Winkel, D. A. Bourgoyne, B. R. Elbing, S. L. Ceccio, M. Perlin, and D. R. Dowling. 2007. "Using cross correlations of turbulent flow-induced ambient vibrations to estimate the structural impulse response. Application to structural health monitoring," The Journal of the Acoustical Society of America, 121(4):1987-1995, doi:10.1121/1.2710463.

12. Huthwaite, P. and F. Simonetti. 2013. "High-resolution guided wave tomography," Wave Motion, 50(5):979-993, doi:10.1016/j.wavemoti.2013.04.004.

13. Druet, T., J.-L. Tastet, B. Chapuis, and E. Moulin. 2019. "Autocalibration method for guided wave tomography with undersampled data," Wave Motion, 89:265-283, doi: 10.1016/j.wavemoti.2019.04.002.

14. Druet, T., T. Hoang, B. Chapuis, and E. Moulin. 2019. "Effect of Fiber Bragg gratings receiver's directivity on guided wave tomography of pipe," in Proceedings of the 12th International Workshop on Structural Health Monitoring, Stanford. 